\title{
A retrospective analysis of the protective efficacy of tafenoquine and mefloquine as prophylactic anti-malarials in non-immune individuals during deployment to a malaria-endemic area
}

\author{
Geoffrey S Dow ${ }^{1 *}$, William F McCarthy ${ }^{1}$, Mark Reid ${ }^{2}$, Bryan Smith ${ }^{1}$, Douglas Tang ${ }^{1}$ and G Dennis Shanks ${ }^{3}$
}

\begin{abstract}
Background: In 2000/2001, the Australian Defense Forces (ADF), in collaboration with SmithKline Beecham and the United States Army, conducted a field trial to evaluate the safety, tolerability and efficacy of tafenoquine and mefloquine/ primaquine for the prophylaxis of malaria amongst non-immune Australian soldiers deployed to East Timor (now called Timor Leste) for peacekeeping operations. The lack of a concurrent placebo control arm prevented an internal estimate of the malaria attack rate and so the protective efficacy of the study regimens was not determined at the time.

Methods: In a retrospective analysis of the trial results, the all species malaria attack rate was estimated for the prophylactic phase of the study which was defined as the period between administration of the first prophylactic dose and the first dose of post-deployment medication. First, the Plasmodium vivax attack rate was estimated during the prophylactic phase of the deployment by adjusting the observed $P$. vivax relapse rate during post-deployment to account for the known anti-relapse efficacies (or effectiveness) of the study medications (determined from prior studies). The all species malaria attack rate ( $P$. vivax and Plasmodium falciparum) was then determined by adjusting the $P$. vivax attack rate based on the ratio of $P$. falciparum to $P$. vivax observed during prior ADF deployments to Timor Leste. This estimated all species malaria attack rate was then used as the 'constant estimated attack rate' in the calculation of the protective efficacy of tafenoquine and mefloquine during the prophylactic phase of the deployment.
\end{abstract}

Results: The estimated attack rate during the prophylactic phase of the study was determined to be $7.88 \%$. The protective efficacies of tafenoquine and mefloquine, with corresponding $95 \%$ confidence intervals $(95 \% \mathrm{Cl})$, were determined to be 100\% (93\%-100\%) and 100\% (79\%-100\%) respectively.

Conclusions: The protective efficacy of tafenoquine (200 mg per day for three days, followed by weekly $200 \mathrm{mg}$ maintenance doses) is similar to that of the weekly standard of care (mefloquine, $250 \mathrm{mg}$ ).

\section{Background}

Tafenoquine is a long elimination half-life [1] primaquine analog that has the potential to replace mefloquine for weekly malaria chemoprophylaxis. Tafenoquine was evaluated in a series of placebo-controlled studies in mixed or semi-immune residents of Africa and Southeast Asia in the late 1990s [2-4]. The weekly standard of care, mefloquine, was also evaluated in some of the same studies. The protective efficacies $(95 \% \mathrm{CI})$ of tafenoquine at the

\footnotetext{
* Correspondence: geoffdow@hotmail.com

'United States Army Medical Materiel Development Activity, 1430 Veterans

Drive, Fort Detrick, Maryland 21702, USA

Full list of author information is available at the end of the article
}

intended dose (200 mg per day for three days followed by weekly $200 \mathrm{mg}$ maintenance doses) and mefloquine in semi-immune residents of Ghana were $86 \%(76-92 \%)$ and $86 \%(72-93 \%)$ respectively [2]. In a second study in semi-immune residents of Kenya, the protective efficacy of tafenoquine was reported to be $86 \%$ (73-93\%) [3]. For a discussion of the use of placebo control arms in malaria chemoprophylaxis studies in which semi-immune individuals are enrolled, see [5].

In $2000 / 2001$, the safety, tolerability and efficacy of weekly tafenoquine and mefloquine were evaluated in malaria non-immune Australian soldiers from the $1^{\text {st }}$ Battalion, Royal Australian Regiment (1RAR) deployed on 
peacekeeping operations to Timor Leste and who volunteered to participate in a Phase 3 study [6]. In that study, which is referred to as Study 033 from here on, the protective efficacy of tafenoquine was not calculated because the study lacked a concurrent placebo control arm. A placebo control arm was not employed in Study 033 since the study volunteers were simultaneously participating in an ongoing peacekeeping operation and a placebo control arm was not considered appropriate in this context because malaria disrupts operational effectiveness. However, the study team concluded that both tafenoquine and mefloquine were effective since no malaria cases were observed during the prophylactic phase of the study, and the observation of Plasmodium vivax relapses during followup, together with epidemiology data from military and civilian sources suggested substantial exposure to malaria [7-10].

The United States Food and Drug Administration (FDA) [11] has stated: "The deployment of military personnel or civilian cohorts to malaria-endemic regions provides an opportunity to study anti-malarial prophylaxis in malarianaive subjects. Since such deployments may last for many months, it is possible to standardize duration of malaria exposure. When placebo-controlled studies cannot be performed, well-characterized epidemiological attack rates can be used to calculate protective efficacy". Furthermore, the FDA [11] also recommends that the calculation of protective efficacy in historical-controlled studies should employ epidemiological attack rates in the study area from at least the past two malaria seasons. In addition, these epidemiological attack rates should closely reflect anticipated attack rates in the study population and should be derived from the same geographical area, during the same seasonal period, with similar rainfall and similar subject exposure.

Malaria epidemiological data were collected from ADF personnel deployed to Timor Leste in the year prior to Study 033 [12]. That study reported an attack rate of $\sim 4.8 \%$ across all deployed military personnel. These data do not completely meet the requirements of the FDA guidance [11] for two reasons. First, the data were collected only for a single year. Second, the operational environment changed between 1999/2000 and 2000/2001, meaning that we do not know whether exposure of ADF personnel to malaria remained constant. Malaria prevalence was measured amongst civilians who were not taking prophylaxis in villages adjacent to garrisoned Australian soldiers during Study 033 [7]. Similar data are not available for the prior year, so cannot be used to directly link exposure in Australian soldiers in the two deployments.

Immunologic markers of malaria exposure continue to be explored as a possible surrogate for observed malaria cases in malaria prophylaxis studies in which a placebo control arm was not used. Antibodies to circumsporozoite protein were measured as a secondary endpoint in the atovaquone-proguanil clinical development program [13]. However, in that study, seroconversion was only $1.1 \%$, and since there were no confirmed symptomatic cases, it is unclear to what extent this reflects true exposure. The US Army recently investigated the possibility that antibodies to merozoite surface protein 1 (MSP1) might serve as a surrogate marker of malaria exposure in non-immune individuals given mefloquine for prophylaxis [14]. Regulatory agencies have not yet embraced the use of such surrogates [11], although it is possible they might if more compelling evidence of their utility became available. In any case, since they are not currently available, a retrospective analysis of stored blood samples, even if feasible, would likely not reveal useful information regarding malaria exposure during Study 033.

Because of the limitations stated above, an alternative approach was needed to satisfy the FDA's requirement that a well-characterized epidemiological attack rate should be used to calculate protective efficacy when a placebo control arm is not appropriate. To this end, the P. vivax attack rate during the prophylactic phase of Study 033 in Timor Leste was estimated through adjustment of the observed post-deployment $P$. vivax relapse rate during the follow-up period of the study to account for the known anti-relapse efficacies (or effectiveness) of the study medications. This methodological separation between what would have been primary $P$. vivax cases during deployment and post-deployment relapses is reasonable, because, while both events are triggered by the same initial exposure, they are independent clinical episodes prevented through independent pharmacologic modalities that pose different risks to the individual and deployed force. An all malaria attack rate was estimated by adjusting the $P$. vivax attack based on the ratio Plasmodium falciparum to $P$. vivax cases observed amongst Australian soldiers in Timor Leste a year prior to Study 033. The estimated all malaria attack rate was then utilized to determine, retrospectively, the protective efficacy of both tafenoquine and mefloquine in Study 033 so a comparison could be made to the placebo controlled studies in semi and mixed immune populations.

\section{Methods}

\section{Definition of prophylactic efficacy}

For the purposes of this study the definition of protective efficacy is the same as was used in the original Study 033 protocol: Ability of study medications with recorded administration to prevent clinical malaria (single positive smear of any species with concurrent signs and symptoms consistent with malaria infection) during prophylactic study drug administration up to and including the first dose of post-deployment medication. 


\section{Definition of anti-relapse efficacy/effectiveness}

For the purposes of this study, anti-relapse efficacy/effectiveness is the degree to which observed (or unsupervised) tafenoquine and primaquine administration can prevent $P$. vivax relapses. There was no distinction made here between the intent or possible timing of 8-aminoquinoline administration, whether it be following a course of suppressive prophylaxis, an additional property of an antimalarial drug also being used in a prophylactic mode, or in sequential combination with a blood schizonticidal drug to treat symptomatic $P$. vivax. In the case of historical estimates of the utility of primaquine, we have used a meta-analysis of effectiveness data [15], since many of the input studies utilized unsupervised primaquine regimens. The corresponding tafenoquine data represent the efficacy of the drug since the studies from which they were derived involved observed use of the drug.

\section{Primary $P$. vivax attacks during deployment and post-deployment relapses are not the same}

Post-deployment $P$. vivax relapses are not the same as primary $P$. vivax attacks occurring during deployment because while triggered by the same initial exposure, are independent clinical events prevented through independent pharmacologic modalities and which pose different risks to the individual and deployed forces.

During a visit to a malarious area, non-immune individuals exposed to $P$. vivax will experience a primary symptomatic attack and if treated with anti-malarial drugs to kill asexual blood stages may still go on to experience one or more hypnozoite-intiated relapses. To prevent such primary attacks during travel or deployment it is conventional to administer a prophylactic that inhibits the development of asexual blood stages or exoerythrocytic stages (or both) in the liver. Since these properties of a prophylactic drug do not prevent the establishment of the $P$. vivax hypnozoite, many individuals who receive such a drug will experience one or more post-deployment relapses rather than a primary attack.

Independent post-deployment administration of primaquine substantially reduces, but not to zero, the number of $P$. vivax relapses due to the effect of the drug on hypnozoites $[15,16]$. This anti-hypnozoite activity is mediated independently of the other effects of primaquine on asexual blood and developing exoerythrocytic stages. The same logic holds for tafenoquine, since the drug exhibits a measurable inhibitory effect on $P$. vivax hypnozoites independent of its other actions on asexual blood stages and developing exoerythrocytic parasites in the liver $[4,17,18]$.

Based on the World War II experience with malaria in the Pacific theatre [19], it seems intuitive that the management of a small number of individuals with $P$. vivax hypnozoites refractory to primaquine (or tafenoquine) is more straight-forward and has less operational impact than a larger number of primary attacks in theatre (in the absence of effective prophylaxis). In contrast, an individual who takes effective prophylaxis without an 8-aminoquinoline is at greater risk of repeated post-deployment $P$. vivax relapses despite having being exposed only once and never experiencing a primary attack. Furthermore, the clinical impact of a primary attack on a traveller may be greater than a relapse in their own country if they do not have access to good medical care while travelling.

Therefore, the deployment prophylactic and anti-relapse efficacies of proposed interventions can and should be measured independently. As a first step towards calculation of the former, an estimate of the attack rate is required. In this study, since the post-deployment relapse rate is known, and the anti-relapse efficacy of the combined study regimens can be estimated, it is possible to estimate the number of individuals exposed to $P$. vivax during a deployment.

\section{Summary of study 033}

From October 2000 through April 2001, the 1RAR was deployed to Timor Leste from Australia for peacekeeping operations [6]. Six hundred and fifty four of these soldiers volunteered to participate in Study 033, and were randomized to receive either tafenoquine $(n=492)$ or mefloquine $(\mathrm{n}=162)$ for malaria prophylaxis. Tafenoquine was administered as a loading dose of $200 \mathrm{mg}$ once per day for three days, followed by weekly $200 \mathrm{mg}$ maintenance doses. Mefloquine was administered as a $250 \mathrm{mg}$ loading dose once per day for three days followed by weekly 250 maintenance doses. During this prophylactic phase of the study, the primary efficacy parameter was the occurrence of microscopically confirmed symptomatic malaria of all species.

Upon return to Australia, subjects randomized to mefloquine and tafenoquine received either $15 \mathrm{mg}$ primaquine twice per day for 14 days (total dose of $420 \mathrm{mg}$ or $5.2 \mathrm{mg} / \mathrm{kg}$ ) or placebo. There was a six month period of follow up. The intent during this phase was to monitor $P$. vivax relapses. Primaquine was given in the mefloquine arm because it is an effective anti-relapse agent and mefloquine is not. A placebo was given in the tafenoquine arm because prior Phase II studies suggested it was also an effective anti-relapse agent.

During the prophylactic phase of the study, there were no symptomatic cases of malaria in either study arm. During the six-month follow-up phase there were four $P$. vivax relapses in the tafenoquine arm and one $P$. vivax relapse in the mefloquine arm.

In the final clinical study report (FCSR) subsequently submitted to the FDA by the Sponsor, the intent to treat (ITT) populations were $n=490$ for tafenoquine, and $n=$ 161 for mefloquine. The ITT denominators were used for the attack rate and protective efficacy calculations 
that follow. The reader should also note that in the FCSR submitted to the FDA by the Sponsor, 21 subjects were reported as being withdrawn from the study. The Sponsor conducted a "worst case analysis" for the intention to treat (ITT) population. Any subject that withdrew during the prophylactic phase was considered a failure (assumed to have had malaria). This "worst case analysis" for the ITT population yielded a protective efficacy, assuming a $100 \%$ attack rate, of $96.5 \%(473 / 490)$ and $97.5 \%(157 / 161)$ for tafenoquine and mefloquine, respectively. The assumption that withdrawn subjects were prophylactic failures is the most conservative approach for such an ITT efficacy analysis. Therefore, an important component of the present study was to confirm that the individuals who withdrew from the study should not be counted as prophylactic failures due to loss to followup. This issue was not explicitly addressed in the original public reporting of the study results [6].

\section{ADF deployments to Timor Leste}

Australian Defense Force infantry battalions commenced peacekeeping operations in Timor Leste in September 1999 as part of International Force East Timor (InterFET) under United Nations Security Resolution 1264 and with Indonesian government agreement. Table 1 summarizes the time period ADF infantry battalions served in Timor Leste up to 30 April 2003. Later battalions starting with the $5^{\text {th }} / 7^{\text {th }}$ Battalion, Royal Australian Regiment (5/7RAR) in October 1999 migrated to operations under the United Nation's Transitional Administration East Timor (UNTAET, Security Resolution 1272 in 1999). In contrast to ADF soldiers under InterFET, those deployed under UNTAET experienced less exposure to night jungle patrols compared to previous operations in multiple regions of Timor Leste; this may have contributed to reduced vector exposure. Vector control operations also improved as lines of communication between Australia and Timor Leste improved; soldiers gradually re-learned basic malaria prevention procedures in infantry units over time.

\section{Documentation of malaria exposure during ADF deployments to Timor Leste}

Malaria is a notifiable disease in all states and territories of Australia under the National Notifiable Disease Surveillance System [20]. Australian Defence Force Health Policy Directive 215 requires notification of malaria infections in military personnel to the state or territory health authority. In addition, all confirmed or suspected cases of malaria in defense personnel or their dependents (whilst on posting to malarious areas) are reported to the Australian Army Malaria Institute (AMI) Central Malaria Registry (CMR). Whenever possible all malaria diagnosis is achieved with light microscopy of thick and thin blood smears. Diagnosis may also be performed using malaria rapid diagnostic tests (RDTs) when accurate diagnosis by microscopy is not available. The notification report to AMI by defence units is accompanied by thick and thin blood films as well as any RDT cards or other devices used to diagnose malaria to allow AMI personnel to verify the diagnosis and species before entry into the CMR.

The CMR was analysed over the period 1 April 1999 to 30 April 2003 for malaria cases in the infantry battalions described in Table 1. Malaria cases were excluded if the infantry soldier had a travel history to another malarious country after leaving Timor Leste and their first confirmed case of malaria. All $P$. vivax relapses were excluded from the analysis irrespective of country of origin. Duplicates entries (total of 17) in the CMR database were confirmed against the original notification and follow-up relapse reports (if applicable) and subsequently discounted. A limitation of the analysis is loss to follow-up of Defense members who leave the ADF and subsequently develop malaria after a military deployment. These cases would be captured by state/territory health authorities but not necessarily by the ADF unless a compensation action was commenced. The analysis was subjected to an independent quality control review. All work complied with the National Statement on Ethical Conduct in Human Research (as amended) guidance on the use of identifiable databanks [21].

\section{Determination of the malaria status of subjects who withdrew from Study 033}

As mentioned earlier, the FCSR report submitted to the FDA by the Sponsor included a "worst case analysis" for the ITT population in which subjects who withdrew from the study were considered prophylactic failures. In the reanalysis of Study 033, there was an attempt to confirm that subjects who withdrew were not in fact prophylactic failures (i.e. did not get malaria) if they remained in the ADF for up to 12 months post their 1 RAR deployment and had no other travel history to malarious areas. Three investigators queried the CMR to determine the malaria status of each of these subjects. As mentioned above, a limitation of the CMR is that it captures malaria cases only for active ADF personnel. Additional ADF records were examined to determine whether any of the subjects withdrawn from the study left the ADF in the twelve months following the end of Study 033.

\section{Observed post-deployment $P$. vivax relapse rate during Study 033}

The number of $P$. vivax cases from study subjects enrolled in Study 033 in the CMR was determined during a period of one year post-deployment. This observation period was longer than that in the Study 033 final clinical study report (6 months) because Chesson strain $P$. vivax 
Table 1 Details of Australian infantry deployments to Timor Leste*

\begin{tabular}{|c|c|c|c|c|c|c|c|c|}
\hline Battalion & Dates & Regions of Timor Leste & Battalion strength & $\begin{array}{l}\text { Months } \\
\text { deployed }\end{array}$ & $\begin{array}{l}\text { Pf attack rate (\%) } \\
\text { Monthly/Cumul *** }\end{array}$ & $\begin{array}{l}\text { Pv attack rate (\%) } \\
\text { Monthly/Cumul }\end{array}$ & $\begin{array}{l}\text { All malaria attack rate } \\
\text { (\%) Monthly/Cumul }\end{array}$ & Prophylaxis \\
\hline $2 \mathrm{RAR}^{* *}$ & Sep 99 through Jan 00 & Dili, Bobanaro district & 681 & 4.33 & $0.31 / 1.32$ & $2.04 / 8.81$ & $2.34 / 10.13$ & Doxycycline 100 mg q.d \\
\hline 3RAR‡ & Sep 99 through Jan 00 & $\begin{array}{l}\text { Dili, Bobanaro district and } \\
\text { Oecussi province }\end{array}$ & 634 & 4.33 & $0.29 / 1.26$ & $2.11 / 9.15$ & $2.59 / 11.2$ & Doxycycline 100 mg q.d \\
\hline 5/7RAR & Nov 99 through Apr 00 & Dili, Bobanaro district & 522 & 6.83 & $0.11 / 0.77$ & $0.81 / 5.56$ & $0.83 / 6.71$ & Doxcycline 100 mg q.d \\
\hline 6RAR & Apr 00 through Oct 00 & Dili, Bobanaro district & 619 & 6.16 & $0.03 / 0.16$ & $0.24 / 1.45$ & $0.26 / 1.62$ & Doxycycline 100 mg q.d \\
\hline 1RAR & Oct 00 through Apr 01 & Dili, Bobanaro district & 723 & 6.00 & $0.00 / 0.00$ & $0.18 / 1.11$ & $0.18 / 1.11$ & $\begin{array}{l}\text { Tafenoquine } 200 \mathrm{mg} \text { or } \\
\text { Mefloquine } 250 \mathrm{mg} \text { weekly }\end{array}$ \\
\hline 4RAR & Apr 01 through Oct 01 & Dili, Bobanaro district & 750 & 7.00 & $0.00 / 0.00$ & $0.08 / 0.53$ & $0.08 / 0.53$ & Mefloquine 250 mg weekly \\
\hline 2RAR & Oct 01 through May 02 & Dili, Bobanaro district & 681 & 6.83 & $0.00 / 0.00$ & $0.11 / 0.73$ & $0.11 / 0.73$ & Mefloquine 250 mg weekly \\
\hline 3RAR & Apr 02 through Oct 02 & Dili, Bobanaro district & 634 & 7.00 & $0.02 / 0.16$ & $0.14 / 0.95$ & $0.18 / 1.26$ & Doxycycline 100 mg q.d \\
\hline 5/7RAR & Oct 02 through Dec 02 & Dili, Bobanaro district & 536 & 2.57 & $0.00 / 0.00$ & $0.28 / 0.71$ & $0.28 / 0.71$ & Doxycycline $100 \mathrm{mg}$ q.d \\
\hline
\end{tabular}

*Values for dates deployed, battalion strength and period deployed are approximate.

**RAR $=$ Royal Australian Regiment.

\#Both 3 RAR and 5/7 RAR soldiers participated in post-exposure prevention studies (Elmes, 2008).

***Monthly attack rate $(\%)=($ Cases/total person-months) $\times 100$; cumulative attack rate $(\%)=($ cases/strength $) \times 100$. 
has been recorded as presenting in the ADF for up to 505 days after the first day of deployment (median 83 days, mean 105 days). As a consequence more $P$. vivax cases were recorded than were reported previously (8 versus 5 ). The 12 month observed post-deployment $P$. vivax attack rate $\left[\mathrm{AR}_{\mathrm{Pv}}\right.$ (post-deployment)] was then calculated as follows (equation 1 ):

$$
\begin{gathered}
A R_{P_{v}}(12 \text { month post-deployment })=\left(\frac{R_{P_{v}}}{N}\right) 100 \% \\
{\left[\left(\frac{8}{651}\right) 100 \%=1.23 \%\right]}
\end{gathered}
$$

Where $\mathrm{R}_{\mathrm{Pv}}=$ total number of $P$. vivax relapses and $\mathrm{N}=$ total ITT sample size

\section{Calculation of the ratio of $P$. falciparum to $P$. vivax cases} during the InterFET deployment (1999/2000)

The ratio of cases of $P$. falciparum to $P$. vivax was estimated as described below and summarized in Table 2 (A-D). The 1RAR soldiers involved in the 2000/01 ADF deployment and who were enrolled in Study 033 arrived in country on 25 October 2000 and returned to Australia on approximately 25 April 2001. This coincided with the wet season and the period of highest seasonal malaria prevalence in Timor Leste [9]. Three separate battalions of Australian soldiers were deployed at times that overlapped this period the year before; Second Battalion, Royal Australian Regimen (2RAR), Third Battalion, Royal Australian Regiment (3RAR) and the 5/7RAR. Their malaria exposure was assumed to be the most relevant because it also coincided with period of highest seasonal malaria prevalence. A fourth, the $6^{\text {th }}$ Battalion, Royal Australian Regiment (6RAR), was deployed in the six months prior to 1RAR in which conditions were drier, and in which the incidence of malaria is usually lower [9]. Together, the 2RAR, 3RAR, and 5/7 RAR were deployed for three periods of overlapping exposure which were relevant. Period 1 was October 25 to October 31 1999, and includes only 2RAR and 3RAR since 5/7 RAR was not yet deployed. Period 2 was from November 1 1999 to January 312000 and included 2RAR, 3RAR and 5/7RAR. Period 3 was from February 12000 to April 25 2000 and includes only 5/7 RAR since the other battalions had returned to Australia.

Table 2A summarizes for each period and regiment, the $P$. falciparum, $P$. vivax, and all species malaria attack rates (AR) calculated as follows (equation 2):

$$
\begin{aligned}
A R(\text { in period })_{P v, P f, \text { all }}= & {\left[\frac{(\text { total cases })_{P v, P f, \text { all }}}{(\text { person-months deployment })}\right] } \\
& \times(\text { months in period }) 100 \%
\end{aligned}
$$

During each of these three time periods, an overall (weighted average) attack rate was calculated with weights based on the number of soldiers in each battalion (Table 2B). Using the overall attack rates (decimal) for each period as estimates of the malaria risk (probability), the estimate of the cumulative 6 month attack rate [((AR

\begin{tabular}{|c|c|c|c|c|}
\hline \multicolumn{5}{|c|}{ A: Cumulative attack rates (\%) by time period, species and battalion exposed during the 1999/2000 ADF deployment* } \\
\hline Time period (months)/(Battalions deployed) & Species & 2RAR $(n=681)$ & 3RAR $(n=634)$ & 5/7RAR $(n=522)$ \\
\hline Period 1: Oct 25 99-Oct 3100 (0.2)/(2RAR and 3RAR) & Pv/Pf/All species & $0.41 / 0.06 / 0.47$ & 0.44/0.07/0.52 & NA/NA/NA \\
\hline Period 2: Nov 1 99-Jan 3100 (3.0)/(2RAR, 3RAR and 5/7 RAR) & Pv/Pf/All species & $6.10 / 0.92 / 7.02$ & $6.56 / 0.98 / 7.76$ & $2.52 / 0.34 / 2.95$ \\
\hline Period 3: Feb 100 - April 2500 (2.8)/(5/7RAR) & Pv/Pf/All species & NA/NA/NA & NA/NA/NA & 2.38/0.32/2.78 \\
\hline
\end{tabular}
(6 month), Table 2C) for each species and all species

Table 2 (A-D): Calculation of the 6 month cumulative attack rate ( $P f, P v$, all species) and $P f$ to $P v$ ratio during the 1999/00 ADF deployment

B: Estimate of overall cumulative attack rate (\%) by period for ALL battalions exposed during the 1999/2000 ADF deployment*

Time period (months)

Period 1: Oct 25 99-Oct 3199 (0.2)

Period 2: Nov 1 99-Jan 31-00 (3.0)

Period 3: Feb 100 - April 2500 (2.8)
Species

$\mathrm{PV} / \mathrm{Pf} / \mathrm{All}$ species

$\mathrm{PV} / \mathrm{Pf} / \mathrm{All}$ species

Pv/Pf/All species
Cumulative attacks for all battalions

$0.42 / 0.06 / 0.49$

$5.24 / 0.77 / 6.12$

2.38/0.32/2.78

C: Estimate of the 6 month cumulative attack rate for battalions exposed during the 1999/00 ADF deployment ${ }^{* *}$

Time period (months)

Periods 1, 2 and 3:Oct 25 99-April 2500 (6.0)
Species

$\mathrm{PV} / \mathrm{Pf} / \mathrm{All}$ species

$0.146(1.15 / 7.89)$

*Overall attack rates are the weighted averages of attack rates for battalions exposed during the period (weights = sample size).

${ }^{* *}$ Cumulative attack rate (probability) $=\mathrm{P} 1+(1-\mathrm{P} 1) * \mathrm{P} 2+(1-\mathrm{P} 1)(1-\mathrm{P} 2) * \mathrm{P} 3$ where $\mathrm{P} 1, \mathrm{P} 2$ and $\mathrm{P} 3$ are the estimated overall attack rates for periods 1,2 and 3 (from Table $\left.2 \mathrm{~B}\right)$.

${ }^{* * *}$ Cumulative $\mathrm{Pf}$ attack for all battalions (from Table 2C)/Cumulative Pv attack rate for all battalions (from Table 2C). 
(P. vivax and $P$. falciparum) were obtained as follows (equation 3$)$ :

$$
\begin{aligned}
A R(\text { six month })_{P v, P f, \text { all }}= & A R_{P 1}+\left(1-A R_{P 1}\right) A R_{P 2} \\
& +\left(1-A R_{P 1}\right)\left(1-A R_{P 2}\right) A R_{P 3}
\end{aligned}
$$

The $P$. falciparum to $P$. vivax ratio $(P f: P v)$ was then determined (Table 2D) as follows (equation 4):

$$
P f: P \nu=\frac{A R(6 \text { month })_{P f}}{A R(6 \text { month })_{P v}} \quad\left[\frac{1.15}{7.89}=0.146\right]
$$

\section{Assumed anti-relapse efficacy/effectiveness of Study 033 regimens}

For estimation of the $P$. vivax attack rate during the prophylactic phase, it was assumed that the anti-relapse efficacy/effectiveness of the combined ( $\left.\mathrm{ARE}_{\text {combined }}\right)$ postexposure prophylaxis regimens (tafenoquine or primaquine) was $82.1 \%$. This was obtained as a weighted average of estimates of the anti-relapse efficacy/effectiveness of tafenoquine and primaquine and was calculated as follows (equation 5):

$$
\begin{aligned}
A R E_{\text {combined }}= & \left(\frac{N_{T q}\left(A R E_{T q}\right)+N_{M e f}\left(A R E_{P q}\right)}{\left(N_{T q}+N_{M e f}\right)}\right) 100 \% \\
& {\left[\left(\frac{490(0.863)+161(0.695)}{(490+161)}\right) 100 \%=82.1 \%\right] }
\end{aligned}
$$

Where $\mathrm{N}_{\mathrm{Tq}}$ and $\mathrm{N}_{\mathrm{Mef}}$ are the ITT sample sizes in the post-exposure tafenoquine and mefloquine arms of Study 033 and $A R E_{T q}$ and $A R E_{P_{q}}$ are the anti-relapse efficacies/effectiveness of tafenoquine and primaquine determined from published studies as outlined in the following paragraphs.

$\mathrm{ARE}_{\mathrm{Pq}}$ was based on pooled data from a number of historical studies in which the relapse rate in a chloroquine only arm $(26.5 \%$; $420 / 1585)$ was compared with chloroquine $+2.5-<5 \mathrm{mg} / \mathrm{kg}$ primaquine $(8.08 \% ; 140 / 1732)$ as reported by [15]. Based on these relapse rates, the assumed $\mathrm{ARE}_{\mathrm{Pq}}$ is $69.5 \%$ ([(0.265-0.0808)/0.265]100\%). Note that this represents the effectiveness of primaquine in this context because administration of the drug in many of the studies analysed by John et al. (2012) was not directly observed.

The assumed anti-relapse efficacy of tafenoquine $\left(\mathrm{ARE}_{\mathrm{Tq}}\right)$ was based on data reported by Walsh et al. [4]. Walsh et al. investigated the prophylactic efficacy of tafenoquine administered as a loading dose of $400 \mathrm{mg} \times 3$, followed by monthly maintenance doses of $400 \mathrm{mg}$ for five months versus placebo in deployed Thai soldiers. $P$. vivax relapses were observed in $3.1 \%(3 / 96)$ of subjects randomized to tafenoquine and completing the study, where as $22.8 \%$ (21/92) of subjects randomized to placebo who completed the study contracted $P$. vivax in the prophylactic phase [4]. In that study, subjects in the placebo control arm who contracted malaria were administered a curative regimen and recruited for a subsequent tafenoquine pharmacokinetic study, meaning that the $P$. vivax relapse rate in this arm is not known. It was assumed the $P$. vivax relapse rate in that study was $100 \%$. Therefore, the assumed anti-relapse rate of tafenoquine was $86.3 \%([(0.228-0.031) / 0.228] 100 \%)$.

\section{Estimated malaria attack rate during Study 033}

The estimated all malaria attack rate during the six-month prophylactic phase of Study 033 was calculated as the sum of the estimated six month attack rates for $P$. vivax and P. falciparum as follows (equation 6).

$$
\begin{aligned}
& A R(\text { prophylaxis phase })_{\text {all }} \\
& \quad=A R_{P v}+A R_{P f} \quad[6.88 \%+1.00 \%=7.88 \%]
\end{aligned}
$$

The calculations are further summarized in Table 3. Note that species other than P. vivax and P. falciparum were excluded.

The $P$. vivax attack rate $\left(\mathrm{AR}_{\mathrm{Pv}}\right)$ during the prophylaxis phase was estimated by adjusting the total observed relapse rate of $1.229 \%$ (8/651) during the post-deployment period to take into account the combined anti-relapse efficacy of $82.1 \%$ in the tafenoquine and mefloquine/ primaquine arms (Eqn 5; Table 3) as follows (equation 7)

$$
\begin{gathered}
A R_{P_{v}}(\text { prophylaxis phase })=\frac{A R_{P v}(12 \text { month post } \text {-deployment })}{1-A R E_{\text {combined }}} \\
{\left[\frac{1.229 \%}{1-0.8213}=6.88 \%\right]}
\end{gathered}
$$

It was assumed that all $P$. vivax cases that would have been observed in the prophylactic phase would also have relapsed in the absence of post-exposure prophylaxis. The observed $P$. vivax relapse rate during the post-deployment period of Study $033(1.229 \%)$ was determined using data from the CMR as described earlier.

The estimate of the $P$. falciparum attack rate during the six-month prophylaxis was based on the estimate of the $P$. vivax attack rate during the prophylaxis phase $(6.88 \%$; from equation 7) and the ratio of $P$. falciparum relative to $P$. vivax (0.146, from equation 4) based on epidemiological data from the 1999/00 ADF deployments to Timor Leste (Table 2) and calculated as follows (equation 8).

$$
\begin{aligned}
A R_{P f}(\text { prophylaxis phase })= & A R_{P v}(P f: P v \text { ratio }) \\
& {[6.88 \%(0.146)=1.00 \%] }
\end{aligned}
$$

\section{Calculation of the protective efficacy and confidence intervals for tafenoquine and mefloquine}

The usual definition of the protective (prophylactic) efficacy $(\mathrm{PE})$ in a placebo-controlled trial was used to determine the 
Table 3 Estimation of malaria attack ( $P v, P f$, all species) during the prophylactic phase (6 month) of Study 033

\begin{tabular}{|c|c|c|}
\hline Data* & Value** & Source \\
\hline Post deployment $P$. vivax relapse rate (\%) amongst Study 033 subjects & 1.23 & $\begin{array}{l}P \vee \text { relapses (8/651) from CMR not original Study } 033 \\
\text { FCSR (Equation 1) }\end{array}$ \\
\hline Anti-relapse effectiveness (\%) of primaquine & 69.5 & Estimated from John et al. 2012 \\
\hline Anti-relapse efficacy (\%) of tafenoquine & 86.3 & Estimated from Walsh et al. 2004b \\
\hline $\begin{array}{l}\text { Anti-relapse efficacy of combined Study } 033 \text { post-exposure } \\
\text { prophylaxis regimens }\end{array}$ & 82.1 & Estimated (Equation 5) \\
\hline$P_{V}$ attack rate (\%) during prophylactic phase of Study 033 & 6.88 & Estimated (Equation 7) \\
\hline Ratio of Pf cases to Pv cases in 1999/2000 ADF deployment (InterFET) & 0.146 & Observed from 1999/2000 ADF deployment (Equation 4) \\
\hline Pf attack rate (\%) during prophylactic phase of Study 033 & 1.00 & Estimated (Equation 8) \\
\hline All malaria attack rate (\%) during prophylactic phase of Study 033 & 7.88 & Estimated (Equation 6) \\
\hline
\end{tabular}

*Observed in Study 033, ADF deployment, assumed from literature or derived. ${ }^{* *}$ Rounded after calculation.

$\mathrm{PE}$ for tafenoquine $\left(\mathrm{PE}_{\mathrm{Tq}}\right)$ during the prophylaxis phase as follows (equation 9):

$$
P E_{T q}=\left(\frac{A R_{P l}-A R_{T q}}{A R_{P l}}\right) 100 \%=\left(1-\frac{A R_{T q}}{A R_{P l}}\right) 100 \%
$$

Where $\mathrm{AR}_{\mathrm{Tq}}$ and $\mathrm{AR}_{\mathrm{Pl}}$ are the cumulative risk estimates (cumulative attack rates) for the period of prophylaxis in those receiving tafenoquine compared to those receiving a hypothetical placebo. A similar definition applies to the protective efficacy of mefloquine $\left(\mathrm{PE}_{\mathrm{Mef}}\right)$. Since Study 033 had no placebo control arm, conventional calculation of protective efficacy and corresponding confidence interval $(\mathrm{CI})$ was not possible. Because in Study 033 there were no cases of malaria it should be noted that, for any estimated attack rate, the point estimate $\mathrm{PE}_{\mathrm{Tq}}$ and $\mathrm{PE}_{\mathrm{Mef}}$ would be $100 \%$. However, standard methods for calculating a confidence interval (CI) for PE (Tq or Mef) depend on the placebo attack rate and sample size. To determine the PE and $\mathrm{CI}$ it was assumed that the attack rate $\left(\mathrm{AR}_{\mathrm{Pl}}\right)$ would have been the estimated all malaria attack rate of $7.88 \%$ calculated above (equation 6) during the prophylactic phase of Study 033. In addition a constant $\mathrm{AR}_{\mathrm{Pl}}$ was (= 7.88\%) used for calculating corresponding $95 \%$ CIs for PE. A sensitivity analysis of this assumption was carried out (Table 4 , see below). In the remainder of this discussion the estimated all malaria attack rate $(=7.88 \%)$ will be referred to as the "estimated $A R$ " $\left(\mathrm{AR}_{\mathrm{Pl}}\right)$.

The attack rate for tafenoquine $\left(A R_{T q}\right)$ and mefloquine $\left(A R_{M e f}\right)$ during the prophylactic phase was determined based on the ITT results of study 033 [0 cases; sample size of 490 (Tq) and 161 (Mef)] and were computed as follows (equation 10):

$$
\begin{aligned}
A R_{T q, M e f}(\text { prophylaxis phase })= & \frac{(\text { All malaria cases })_{T q, M e f}}{N_{T q, M e f}} \\
& {\left[\frac{0}{490}, \frac{0}{161}\right] }
\end{aligned}
$$

Table 4 Effect of using a constant baseline attack rate on the precision (confidence interval) of the estimated protective efficacy of tafenoquine compared to assuming

\begin{tabular}{|c|c|c|c|}
\hline $\mathrm{AR}_{\mathrm{PI}}(\text { cases } / \mathrm{n})^{1}$ & $\mathrm{AR}_{\mathrm{Tq}}(\text { cases } / \mathrm{n})^{2}$ & $\mathrm{PE}_{\mathrm{Tq}}$ & $\mathrm{LL}\left(\mathrm{PE}_{\mathrm{Tq}}\right)^{3}$ \\
\hline $1 \%(1 / 100)$ & $0(0 / 490)$ & $100 \%$ & $45 \%(44.87)$ \\
\hline 1\% (10/1000) & $0(0 / 490)$ & $100 \%$ & $45 \%(44.91)$ \\
\hline $1 \%{\text { (constant })^{4}}^{4}$ & $0(0 / 490)$ & $100 \%$ & $44 \%(44.10)$ \\
\hline $2 \%(1 / 50)$ & $0(0 / 490)$ & $100 \%$ & $72 \%(72.43)$ \\
\hline $2 \%(2 / 100)$ & $0(0 / 490)$ & $100 \%$ & $72 \%(72.46)$ \\
\hline $2 \%{\text { (constant })^{4}}^{4}$ & $0(0 / 490)$ & $100 \%$ & $73 \%(72.54)$ \\
\hline $3 \%(3 / 100)$ & $0(0 / 490)$ & $100 \%$ & $82 \%(81.65)$ \\
\hline $3 \%(30 / 1000)$ & $0(0 / 490)$ & $100 \%$ & $82 \%(81.69)$ \\
\hline $3 \%$ (constant) $^{4}$ & $0(0 / 490)$ & $100 \%$ & $82 \%(81.70)$ \\
\hline $5 \%(1 / 20)$ & $0(0 / 490)$ & $100 \%$ & $89 \%(88.97)$ \\
\hline $5 \%(2 / 40)$ & $0(0 / 490)$ & $100 \%$ & 89\% (88.98) \\
\hline $5 \%(5 / 100)$ & $0(0 / 490)$ & $100 \%$ & $89 \%$ (88.99) \\
\hline $5 \%(50 / 1000)$ & $0(0 / 490)$ & $100 \%$ & $89 \%$ (89.02) \\
\hline $5 \%{\text { (constant })^{4}}^{4}$ & $0(0 / 490)$ & $100 \%$ & $89 \%(89.02)$ \\
\hline $10 \%(1 / 10)$ & $0(0 / 490)$ & $100 \%$ & $94 \%$ (94.49) \\
\hline $10 \%(5 / 50)$ & $0(0 / 490)$ & $100 \%$ & $95 \%(94.50)$ \\
\hline $10 \%(10 / 100)$ & $0(0 / 490)$ & $100 \%$ & $95 \%$ (94.50) \\
\hline $10 \%(100 / 1000)$ & $0(0 / 490)$ & $100 \%$ & $95 \%(94.51)$ \\
\hline $10 \%$ (constant) $^{4}$ & $0(0 / 490)$ & $100 \%$ & $95 \%(94.51)$ \\
\hline
\end{tabular}
an attack rate based on results from hypothetical placebo controlled trials with different sample sizes

${ }^{1}$ Placebo attack rate based on hypothetical trial results (cases/n) or assumed to be a known constant value (no variability).

${ }^{2}$ Observed attack rate for tafenoquine in Study 033.

${ }^{3}$ Lower limit of the one-side $95 \% \mathrm{Cl}$ for the protective efficacy of tafenoquine [LL(PETq)]. Koopman's (score test) method was used when the placebo attack rate $\left(A R_{P I}\right)$ is based on hypothetical placebo results (cases/n). StatXact (vs.9.0) was used for calculations and the confidence level was set to $90 \%$ [for ARTq $=0$ $(0 / n)$ this gives a lower limit of a one-sided $95 \%$ interval for $\left.P E_{T q}\right]$. ${ }^{4}$ When $\mathrm{AR}_{\mathrm{PI}}$ is assumed to be a known constant equation 11 (bottom) was used to obtain $\mathrm{LL}\left(\mathrm{PE}_{\mathrm{Tq}}\right)$. 
The 95\% CI for $\mathrm{PE}_{\mathrm{Tq}}\left(\mathrm{PE}_{\mathrm{Mef}}\right)$ was obtained by first computing the lower and upper limits (LL, UL) for a proportion $\left(\mathrm{AR}_{\mathrm{Tq}}\right.$ or $\left.\mathrm{AR}_{\mathrm{Mef}}\right)$ using the Wilson (score) method, and then converting these limits into corresponding limits for $\mathrm{PE}$ (assuming $\mathrm{AR}_{\mathrm{Pl}}=7.88 \%$ ). When there are 0 cases, $\mathrm{AR}=0(0 / \mathrm{n})$ and the lower confidence limit for AR is always 0 and the corresponding upper limit for PE is $100 \%$. Given lower and upper Wilson confidence limits for the attack rates $\left(\mathrm{LL}_{\mathrm{AR}}, \mathrm{UL}_{\mathrm{AR}}\right)$ the corresponding limits for $\mathrm{PE}$ (Tq or Mef) are therefore given as follows (equation 11):

$$
\begin{aligned}
& L L_{P E}=\left(1-\frac{U L_{A R}}{0.0788}\right) 100 \% \\
& U L_{P E}=\left(1-\frac{L L_{A R}}{0.0788}\right) 100 \%\left[\left(1-\frac{0}{0.0788}\right) 100 \%=100 \%\right]
\end{aligned}
$$

The above method for determining a confidence interval for PE assumes a constant estimated AR (7.88\%). This assumption does not take into account additional variability had the malaria exposure during Study 033 been estimated based on results from a placebo control arm (with a given sample size). A sensitivity analysis was conducted (Table 4) to determine the effect of assuming a constant estimated attack rate on the resulting 95\% lower confidence limit for the protective efficacy of tafenoquine $\left[\mathrm{LL}\left(\mathrm{PE}_{\mathrm{Tq}}\right)\right]$. Hypothetical estimated attack rates $\left(\mathrm{AR}_{\mathrm{Pl}}\right)$ from $1 \%-10 \%$ and sample sizes $\left(\mathrm{n}_{\mathrm{PL}}\right)$ from $10-$ 1000 were considered. Combinations of $A_{P l}$ and $n_{P l}$ were selected to give integer values for the corresponding number of placebo cases $\left(n_{P L} \times A_{P L}\right)$. All calculations assumed no observed cases in the tafenoquine arm and a sample size (ITT) of $\mathrm{n}=490$ (Study 033 results). Over the range of $\mathrm{AR}_{\mathrm{Pl}}$ considered (1\%-10\%) the estimate of the lower limit of the $95 \%$ confidence interval (one-sided) for $\mathrm{PE}_{\mathrm{Tq}}$ did not vary substantially with changes in sample size $(<1 \%$ change, see Table 4$)$. The minimal effect of sample size on $\mathrm{LL}\left(\mathrm{PE}_{\mathrm{Tq}}\right)$ is because there were no observed malaria cases and the sample size in the tafenoquine arm employed in Study 033 was large (ITT $n=490$ ). For a given $\mathrm{AR}_{\mathrm{Pl}}$ and $\mathrm{n}_{\mathrm{Pl}}$, Koopman's (score test) method was used for computing the lower 95\% limit for PE. StatXact (vs. 9.0) was used and the confidence level was set to $90 \%$ (gives lower limit of a one-sided 95\% interval and is the convention used when the point estimate for $\mathrm{PE}=100 \%)$.

\section{Results}

The observed attack rates for P. falciparum and P. vivax are tabulated for each ADF deployment in Table 1. All malaria species attack rates for 2RAR, 3RAR and 5/7 RAR were $10.13 \%, 11.20 \%$ and $6.71 \%$, respectively (Table 1 ). All species attack rates were substantially lower for subsequent deployments (including 1 RAR), ranging from 0.53 -
$1.62 \%$ (Table 1). The aggregate $P$. falciparum and $P$. vivax attack rates during the 1999/2000 deployments were $7.88 \%$ and $1.15 \%$, yielding a $P$. falciparum to $P$. vivax species ratio of 0.146 (Table 2). For the 1 RAR deployment from 25 October 2000 to 25 April 2001, eight post-deployment $P$. vivax cases were observed amongst a deployed population of 723, yielding an observed post-deployment relapse rate of $1.11 \%$.

All eight $P$. vivax cases following the 1RAR deployment were amongst individuals recruited for Study 033 (note that not all 723 1RAR soldiers were recruited for Study 033). Thus, the observed post-deployment $P$. vivax relapse rate during Study 033 was $1.23 \%$ (8/651). The number of $P$. vivax relapses observed (8) was higher than reported previously [6]. This was a consequence of extending the observation period from six months (per the study design) to 12 months due to additional data reported to the ADF's CMR. The all malaria attack rate during the prophylactic phase of Study 033 was estimated to be $7.88 \%$ (see summary in Table 3 ). The CMR contained no records of malaria cases amongst 20 of 21 withdrawn subjects who remained in the ADF for twelve months following Study 033. A single withdrawn subject, randomized to the mefloquine arm, remained in the ADF for 11 months following Study 033. The CMR also contained no record of that subject having contracted malaria during this period.

In Study 033, of 490 ITT subjects given tafenoquine and 161 ITT subjects given mefloquine, no symptomatic cases of malaria were observed during the prophylactic phase ([6], see Table 5). None of the subjects listed as withdrawn from the study in the FCSR were found to have contracted malaria during the prophylactic phase. Therefore, the protective efficacy of both regimens was $100 \%$. Utilizing the all species malaria attack rate of $7.88 \%$ during the prophylactic phase of the study as the estimated attack rate, the lower limits of the 95\% confidence interval (one-sided, as outlined in Methods) for the protective efficacy of tafenoquine and mefloquine were 93\% and $79 \%$, respectively (Table 5 ).

\section{Discussion}

This paper describes methodology for calculating protective efficacy when there is no concurrent placebo control arm data. Using this methodology, it was possible to demonstrate that tafenoquine exhibited a protective efficacy of $100 \%$ with a $95 \%$ lower confidence limit of $93 \%$ while mefloquine exhibited a protective efficacy of $100 \%$ with a $95 \%$ lower confidence limit of $79 \%$. The lower value of the lower limit for mefloquine is due to the smaller sample size (161 vs 490). These calculations are based on a retrospective estimate of an attack rate of $7.88 \%$. That attack rate was calculated based on the following assumptions: (i) that the P. falciparum:P. vivax species ratio in 
Table 5 Protective efficacy (and confidence interval) for mefloquine and tafenoquine for malaria prophylaxis in non-immune Australian soldiers deployed to Timor Leste for six months in 2000/2001 (an attack rate of 7.88\% was assumed)

\begin{tabular}{|c|c|c|c|c|c|c|}
\hline & $\begin{array}{l}\text { Results during the prophylactic } \\
\text { phase of Study } 033\end{array}$ & $\begin{array}{l}\text { Protective efficacy (PE) during } \\
\text { prophylactic phase of Study } 033\end{array}$ & & & & \\
\hline Drug & ITT Population & Malaria cases & Subjects lost to follow-up & $\begin{array}{l}\text { Drug attack rate } \\
\text { (\%) }(95 \% \mathrm{Cl})^{*}\end{array}$ & $P E(\%)^{* *}$ & $95 \% \mathrm{Cl}^{* * *}$ \\
\hline Mefloquine & 161 & 0 & 0 & $0(0,0.549)$ & 100 & $79-100$ \\
\hline Tafenoquine & 490 & 0 & 0 & $0(0,1.653)$ & 100 & $93-100$ \\
\hline
\end{tabular}

${ }^{*}$ Confidence interval $(\mathrm{Cl})$ for the attack rate (AR) is based on Wilson (score) method for proportions. In the special case when $A R=0$ ( $\left.0 / n\right)$, the upper limit corresponds to a one-sided upper $95 \%$ limit. For $A R=0$, the lower limit is set to zero. **PE $=[1$ - drug $A R / 0.0788)] 100$. For zero cases, $P E=100 \%$.

***95\% Cl for protective efficacy (PE) obtained from corresponding limits for the drug attack rates. The lower limit for PE $=[1-\mathrm{AR}$ upper limit/0.0788] $\mathrm{x} 100$. The upper AR limits for tafenoquine (0/490) and mefloquine (0/161) are $0.549 \%$ and $1.653 \%$. For PE $=100 \%$, the upper limit is set to $100 \%$.

the Study 033 was the same as that directly observed in Australian soldiers in the 1999/2000 deployment, (ii) that the anti-relapse effectiveness of primaquine at the dose used in Study 033 was 69.5\%, and (iii) that the anti-relapse efficacy of the tafenoquine dose used in Study 033 was $86.3 \%$. These assumptions are conservative for the reasons outlined in the following paragraphs. Therefore, the attack rate reported in this study is likely to be an under-estimate.

The utilization of a P. falciparum:P. vivax ratio based on observed cases in Australian soldiers in 1999/2000 is the most conservative of the reasonable alternatives. It could have been assumed that there was no $P$. falciparum malaria during the 1 RAR deployment, since none was directly observed. Alternatively, the higher P. falciparum: $P$. vivax ratios observed in the unprophylaxed civilian population living close to garrisoned Australia soldiers during Study $033(\sim 1: 1)$ or the $\sim 2: 1$ ratio observed for malaria cases originating amongst Australian soldiers in Timor Leste during the 1999-2000 deployment [7,12] could have been used. Assuming no exposure to $P$. falciparum would not have been reasonable given that there was clearly transmission of $P$. falciparum amongst the civilian population living close to the 1 RAR garrison positions [7]. Assuming P. falciparum:P. vivax ratios of $1: 1$ or $2: 1$ would imply attack rates of $14 \%$ and $22 \%$ respectively. However, this would have assumed either parity in malaria exposure between unprophylaxed civilians and soldiers, or that compliance with doxycycline and primaquine prophylaxis could be estimated in the absence of data (remember that in Study 033 compliance with medications was recorded whereas in the 1999-2000 deployment it was not). While these assumptions may be defensible, the more conservative of the reasonable approaches was taken.

Using data from John et al. [15], it was assumed that the anti-relapse efficacy of the primaquine regimen used in Study 033 was $69.5 \%$. This is likely to have been an underestimate since the dose used in Study $033(5.2 \mathrm{mg} / \mathrm{kg})$ is higher than that $(2.5-<5 \mathrm{mg} / \mathrm{kg})$ for which John et al. provide data. It was decided to use effectiveness data for low dose primaquine $(2.5-<5 \mathrm{mg} / \mathrm{kg})$ because there are only two studies in the literature which have compared relapse rates for a blood schizonticidal drug with and without high dose primaquine (>5 mg/kg). Neither of these is directly analogous to Study 033. Baird et al. [22] reported that the anti-relapse efficacy of $10 \mathrm{mg} / \mathrm{kg}$ primaquine in combination with a standard chloroquine regimen in Irian Jaya was $82.3 \%$ relative to chloroquine alone after 28 days follow-up. However, the higher dose $(10 \mathrm{mg} / \mathrm{kg}$ v $5.2 \mathrm{mg} / \mathrm{kg}$ in Study 033), short follow-up period (one month versus 12 months in Study 033) and the presence of chloroquine-resistance $P$. vivax in Irian Jaya (recurrences may be recrudescences not true relapses) are confounding. Leslie et al. [23] reported a higher antirelapse efficacy for high dose primaquine in Pakistan. However, reliance on a single study from South Asia would have been inappropriate since recurrence rates following primaquine administration may be lower there than in South East Asia.

The assumed anti-relapse efficacy of tafenoquine of 86.3\% was calculated using data for a prophylactic regimen of tafenoquine that was different from that used in Study 033 [4]. This is likely to have been an under-estimate since pharmacokinetic modeling studies suggest that this regimen (400 mg per day for three days followed by monthly maintenance doses) generates lower overall exposure levels than the Study 033 regimen despite the higher loading dose (Dow et al., unpublished observations). The calculations also assume similar $P$. vivax relapse rates and susceptibility to tafenoquine in Thailand and Timor Leste. This is reasonable given the long follow-up times in both the Walsh study and Study 033, and the lack of evidence suggesting any regional differences in susceptibility to 8 -aminoquinolines in the western Pacific.

Mefloquine is an effective anti-malarial drug and remains the standard of care for weekly malaria prophylaxis where this is justified by the risk:benefit context. The reported efficacy of mefloquine prophylaxis in malaria naïve individuals is broadly similar to the efficacy of tafenoquine reported in this and prior clinical reports. In U.S. Peace Corps volunteers stationed in East 
Africa, where the majority of $P$. falciparum strains were chloroquine-resistant, the protective efficacy $(95 \% \mathrm{CI})$ of mefloquine prophylaxis relative to chloroquine and chloroquine/proguanil prophylaxis was $94 \%$ (86-97\%) and $86 \%$ (67-94\%) respectively [24]. Presumably these also represent under-estimates of the true efficacy of mefloquine because both chloroquine and chloroquine-proguanil presumably have greater efficacy than placebo. In European tourists travelling to East Africa, the protective efficacy of mefloquine prophylaxis relative to no malaria prophylaxis was $94 \%$ [25].

The protective efficacy of tafenoquine and mefloquine during the deployment period in Study 033 was 100\% since no symptomatic cases of malaria were observed. This was lower than the level of prophylactic efficacy of the same drugs ( $\sim 86 \%$ for both drugs) observed amongst semi-immune residents of Ghana in one of the Phase II studies [2]. This seems counter-intuitive given the conventional wisdom that prophylactic anti-malarials should be more effective in non-immunes than semi-immunes due to the presumably enhancing effect of immunity in the latter group. However, it is not known whether this hypothesis is correct and there has not been a systematic review of the literature addressing this question. Also, there is a paucity of placebo-controlled field studies that have definitively determined the prophylactic efficacy of approved regimens in non-immune travellers since these are very challenging to perform. In the specific case of the Ghana [2] and Study 033, it is reasonable to speculate that the apparent differences in efficacy may be due to false positive microscopy. Even in a high attack rate setting, small rates of false positive microscopy $(<1 \%)$ may result in an underestimate of true prophylactic efficacy that is of the order of magnitude of the difference in efficacy observed between the Ghana and Study 033 [26]. Furthermore, in a study involving non-immunes, a true malaria case, whether correctly or incorrectly diagnosed using microscopy, is likely to be recognized as such by the study team because it will be symptomatic. This is less likely to be the case in semi-immunes where it is routine to detect parasites by microscopy in individuals who are asymptomatic.

The conclusion that prophylactic efficacy of tafenoquine and mefloquine was $100 \%$ in Study 033 applies only to the period of time between the first dose of the drug and the first dose of post-deployment medication. In the case of mefloquine the persisting risk of a postdeployment case of $P$. falciparum malaria from a late deployment exposure is managed through administration of additional weekly doses of the drug for four weeks, while the risk of $P$. vivax relapses is managed through administration of primaquine (as in Study 033). In the case of tafenoquine, it is known that symptomatic failures occur when plasma levels of the drug fall below
$80 \mathrm{ng} / \mathrm{ml}$ [27]. Therefore, it is anticipated that additional administration of tafenoquine will be required postdeployment to manage the residual risk of $P$. falciparum cases, although the precise regimen has not been determined. Based on open-label clinical studies [16], a residual risk of post-deployment $P$. vivax relapses of approximately the same magnitude as primaquine can be anticipated.

The population that may benefit the most from drugs for malaria prophylaxis are non-immune individuals deployed or travelling to areas with endemic malaria, although in some cases prophylaxis may also have considerable utility for malaria control efforts in endemic countries. In the context of a clinical study to determine the protective efficacy of a new prophylactic anti-malarial in non-immune individuals, it may be very challenging to directly determine an attack rate if the use of a placebo is not feasible. During a military engagement, placebo control arms are generally not used because of their possible impact on mission effectiveness. As argued elsewhere [5], the use of placebo in nonimmune individuals more generally is acceptable if it does not expose study participants to a substantial risk of severe illness or death. This is feasible in Phase I challenge studies but may not be in a deployed setting for military or civilian participants. In the specific context of a prophylaxis study involving a deploying military force, if preparations for execution of the study are conducted rapidly there may be insufficient time to adequately document malaria exposure for two prior years as required by the FDA guidance [11]. One approach to determining a well-characterized attack rate would be to radically cure a cohort of civilians resident in the same area, and prospectively document new infections. Necessarily this assumes that civilian and military exposure to malaria is the same. The method described here, with different inherent assumptions, whereby an attack rate during the prophylactic phase is estimated based on adjustment of the observed post-deployment $P$. vivax relapse rate during follow-up to account for the anti-relapse efficacy of the study drugs, is an alternative approach that could be considered.

\section{Conclusions}

This study retrospectively determined a conservative estimate of $7.88 \%$ for the all species malaria attack rate to which Australian Defence Forces personnel on peacekeeping duties in Timor Leste were exposed during their participation in a Phase III prophylaxis study to evaluate the prophylactic efficacy of tafenoquine and mefloquine. The prophylactic efficacy of mefloquine and tafenoquine during the period between administration of the first prophylactic dose and the first dose of post deployment medication was $100 \%$ (93-100\%) and 100\% (79-100\%) respectively. The methodology employed to determine an attack retrospectively in the absence of placebo 
control arm should be considered when planning future prophylactic studies involving malaria naïve subjects in which it is not feasible to include a placebo control arm.

\section{Competing interests}

The authors have a personal and professional interest in ensuring the regulatory approval of tafenoquine for malaria prophylaxis but no competing interests.

\section{Authors' contributions}

GSD led the technical team that performed the analysis and wrote the manuscript. MR extracted records from the Central Malaria Registry and conducted the analysis of attack rates from prior deployments. WFM and DT performed the statistical analyses. BS and DS provided valuable perspective on the clinical aspects of malaria, methodological aspects of clinical trial and product history with tafenoquine. All authors read and approved the final manuscript.

\section{Acknowledgments}

We wish to acknowledge the assistance of Mr Kenneth Lilley with data retrieval from the Central Malaria Registry and SGT John Humphreys for operational research into the infantry battalion movements and timelines during InterFET and UNTAET operations in Timor Leste. We are grateful to current and former colleagues at NIH, USAMMDA, WRAIR, GSK and AMI, in particular, Dr Mike Edstein and Dr Peter Nasveld, for their perspective and advice regarding the concepts articulated in this manuscript. This study would not have been feasible without the participation of members of $1^{\text {st }}$ Battalion, Royal Australian Regiment, in Study 033. We wish to acknowledge the efforts of the original tafenoquine study team including Leonard Brennan, Ivor Harris, Scott Kitchener, Peter Leggat, Karl Rieckmann, Bob Cooper, Stephen Frances, Michael Reid, Alyson Auliff, Bruce Russell, Stephen McLeod-Robertson, John Staley, Kerryn Rowcliffe, John Ross, Brian Potter, Mike Edstein and Peter Nasveld from the ADF, Philip Pickford, Caron Kerr, Keith Barker and Dominic Galvin from GlaxoSmithKline Research \& Development Limited, Colin Ohrt from the Walter Reed Institute of Research, and William Prescott and Ann Aultman from the US Army Medical Materiel Development Activity.

\section{Disclaimer}

The opinions expressed are those of the authors and do not necessarily reflect those of the Australian Defence Force or the U.S Department of Defense.

\section{Author details}

'United States Army Medical Materiel Development Activity, 1430 Veterans Drive, Fort Detrick, Maryland 21702, USA. ${ }^{2}$ Clinical Network Services, 4/88 Jephson Street, Toowong, Queensland 4066, Australia. ${ }^{3}$ Australian Army Malaria Institute, Weary Dunlop Drive, Gallipoli Barracks, Enoggera, Queensland 4051, Australia.

Received: 27 August 2013 Accepted: 20 January 2014 Published: 6 February 2014

\section{References}

1. Charles BG, Miller AL, Nasveld PE, Reid MG, Harris IE, Edstein MD: Population pharmacokinetics of tafenoquine during malaria prophylaxis in healthy subjects. Antmicrob Agents Chemother 2007, 51:2709-2715.

2. Hale BR, Owusu-Agyei S, Fryauff DJ, Koram KA, Adjuik M, Oduro AR, Prescott WR, Baird JK, Nkurmah F, Ritchie TL, Franke ED, Binka FN, Horton J, Hoffman SL: A randomized, double-blind, placebo-controlled, dose-ranging trial of tafenoquine for weekly prophylaxis against Plasmodium falciparum. Clin Infect Dis 2003, 36:541-549.

3. Shanks GD, Oloo AJ, Aleman GM, Ohrt C, Klotz FW, Braitman D, Horton J, Brueckner R: A new primaquine analogue, tafenoquine (WR 238605), for prophylaxis against Plasmodium falciparum malaria. Clin Infect Dis 2001, 33:1968-1974.

4. Walsh DS, Eamsila C, Sasiprapha T, Sangkharomya S, Khaewsathien P, Supakalin P, Tang DB, Jarasrumsichol P, Cherdchu C, Edstein MD, Rieckmann KH, Brewer TG: Efficacy of monthly tafenoquine for prophylaxis of Plasmodium vivax and multi-drug resistant $P$. falciparum malaria. J Infect Dis 2004, 190:1456-1463.

5. Dow GD, Magill AJ, Ohrt C: Clinical development of new prophylactic antimalarial drugs after the $5^{\text {th }}$ Amendment to the Declaration of Helsinki. Ther Clin Risk Manag 2008, 4:803-819.

6. Nasveld PE, Edstein MD, Reid M, Brennan L, Harris IE, Kitchener SJ, Leggat PA Pickford P, Kerr C, Ohrt C, Prescott W, Tafenoquine Study Team: Randomized, double-blind study of the safety, tolerability and efficacy of tafenoquine versus mefloquine for malaria prophylaxis in non-immune subjects. Antimicrob Agents Chemother 2010, 54:792-798.

7. Bragonier R, Reyburn H, Nasveld P, Edstein M, Auliffe A: Rainy-season malaria prevalence in Bobonaro district, East Timor. Ann Trop Med Parasitol 2002, 96:739-743.

8. Kolaczinski J, Webster J: Malaria control in complex emergencies: the example of East Timor. Trop Med Int Health 2003, 8:48-55.

9. Martins JA, Zwi AB, Martins N, Kelly PM: Malaria control in Timor-Leste during a period of political instability: what lessons can be learned? Conflict and Health 2009, 3:11.

10. WHO: "Weekly International Health Bulletin for East Timor, 1999 Week 44 - 2001 Week 39. Geneva: WHO; 1999.

11. FDA: Draft Guidance for Industry - Malaria: Developing drugs and non-vaccine biological products for treatment and prophylaxis. Washington DC: United States Department of Health and Human Services; 2007.

12. Kitchener SJ, Auliff AM, Rieckmann KH: Malaria in the Australian Defence Force during and after participation in the International Force in East Timor (INTERFET). Med J Aust 2000, 173:583-585.

13. Overbosch D, Schilthuis $H$, Bienzle U, Behrens RH, Kain KC, Clarke PD, Toovey S, Knobloch J, Nothdurft HD, Shaw D, Roskell NS, Chulay JD, Malarone International Study Team: Atovaquone-proguanil versus mefloquine for malaria prophylaxis in non-immune travelers: results from a randomized, double-blind study. Clin Infect Dis 2001, 33:1015-1021.

14. Moon JE, Deye GA, Miller L, Fracisco S, Miller RS, Tosh D, Cummings JF, Ohrt C, Magill AJ: Plasmodium falciparum infection during suppressive prophylaxis with mefloquine does not induce an antibody response to merozoite surface protein-1(42). Am J Trop Med Hyg 2011, 84:825-829

15. John GK, Douglas NM, von Seidlein L, Nosten F, Baird JK, White NJ, Price RN: Primaquine radical cure of Plasmodium vivax: a critical review of the literature. Malar J 2012, 11:280

16. Elmes NJ, Nasveld PE, Kitchener SJ, Kocisko DA, Edstein MD: The efficacy and tolerability of three different regimens of tafenoquine versus primaquine for post-exposure of Plasmodium vivax malaria in the Southwest Pacific. Trans R Soc Trop Med Hyg 2008, 102:1095-1101.

17. Dow GS, Gettayacamin M, Hansukjariya P, Imerbsin R, Komcharoen $S$, Sattabongkot J, Kyle D, Milhous W, Kenworthy D, Miller A, Veazey J, Ohrt C: Radical curative efficacy of tafenoquine combination regimens in Plasmodium cynomolgi-infected Rhesus monkeys (Macaca mulatta). Malar J 2011, 20:212

18. Peters W, Robinson BL, Milhous WK: The chemotherapy of rodent malaria, LI. Studies on a new 8-aminoquinoline, WR 238,605. Ann Trop Med Parasitol 1993, 87:547-552.

19. Joy RJT: Malaria in American troops in the south and Southwest pacific in world War II. Medical History 1999, 43:192-207.

20. Australian Government Department of Health: Introduction to the national notifiable disease surveillance system. https://www.health.gov.au/internet/ main/publishing.nsf/Content/cda-surveil-nndssnndssintro.htm.

21. Australian Government National Health and Medical Research Council: National statement on ethical conduct in human research (2007) - updated December 2013. http://www.nhmrc.gov.au/guidelines/publications/e72.

22. Baird JK, Basri H, Subianto B, Fryauff DJ, McElroy PD, Lekasana B, Ritchie TL, Masbar S, Wignall FS, Hoffman SL: Treatment of chloroquine-resistant Plasmodium vivax with chloroquine and primaquine or halofantrine. J Infect Dis 1995, 171:1678-1682.

23. Leslie T, Mayan I, Mohammed B, Erasmus P, Kolaczinski J, Whitby CJ, Rowland M: A randomized trial of an eight week, once-weekly primaquine regimen to prevent relapse of Plasmodium vivax in Northwest Frontier Province, Pakistan. PLoS One 2008, 3:e2861.

24. Lobel HO, Miani M, Eng T, Bernard KW, Hightower AW, Campbell CC: Long-term malaria prophylaxis with weekly mefloquine. Lancet 1993, 341:848-51. 
25. Steffen R, Fuchs E, Schildknecht J, Naef U, Funk M, Schlagenhauf $P$, Phillips-Howard P, Nevill C, Sturchler D: Mefloquine compared with other malaria chemoprophylactic regimens in tourist visiting east Africa. Lancet 1993, 341:1299-303.

26. Ohrt C, Purnomo, Sutamihardja MA, Tang D, Kain KC: Impact of microscopy error on estimates of protective efficacy in malaria-prevention trials. $J$ Infect Dis 2002, 186:540-546.

27. Edstein MD, Kocisko DA, Eamsila C, Charles BG, Rieckmann KH: Plasma concentrations of tafenoquine, a new long-acting antimalarial agent, in Thai soldiers receiving monthly prophylaxis. Clin Infect Dis 2003,

37:1654-1658.

doi:10.1186/1475-2875-13-49

Cite this article as: Dow et al:: A retrospective analysis of the protective efficacy of tafenoquine and mefloquine as prophylactic anti-malarials in non-immune individuals during deployment to a malaria-endemic area. Malaria Journal 2014 13:49

\section{Submit your next manuscript to BioMed Central and take full advantage of:}

- Convenient online submission

- Thorough peer review

- No space constraints or color figure charges

- Immediate publication on acceptance

- Inclusion in PubMed, CAS, Scopus and Google Scholar

- Research which is freely available for redistribution 